\title{
Non-Monotonic NPI-Licensing, Definite Descriptions, and Grammaticalized Implicatures
}

\author{
Daniel Rothschild \\ Princeton University
}

\section{Non-monotonic Contexts and Negative Polarity Items}

This paper comes in two parts: the first argues for the thesis that, in English, nonmonotonic contexts generally license NPIs and that standard extensions of the notion of downward-entailment do not adequately explain this. The second part argues that this observation puts pressure on some accounts of scalar implicatures and singular definite descriptions.

A downward-entailing context has the property that the replacement of the predicate in the context by a stronger predicate preserves truth. So, for instance, presuppositions aside, the context after "every" in (1) where the NPI "ever" appears is downward entailing.

(1) Every person who'd ever been to the bank got infected.

It is common ground that weak NPIs, which in English includes "ever," "at all" and non-free-choice "any," are allowed in downward-entailing contexts-intervention effects and locality conditions aside. ${ }^{1}$

What is controversial is the status of various environments that, on the surface, appear to be non-monotonic rather than downward-entailing. In English, almost all of these environments license NPIs. ${ }^{2}$ I will discuss three proposals for understanding the rather loose licensing conditions of NPIs and argue that none of them adequately capture the facts of non-monotonic licensing. I will suggest that the best account is simply to say that NPIs are licensed in non-monotonic as well as downward-entailing environments, and I will defend this generalization against apparent exceptions.

First, here are an assortment of cases where apparently non-monotonic contexts allow NPIs. All I mean by "apparently non-monotonic" is that in each case, intuitively, neither a stronger nor a weaker predicate can replace the original predicate and preserve truth. I do not think all these contexts license NPIs for exactly

Many thanks to Eliza Block, Jessica Boyd, Richard Breheny, Kai von Fintel, Nathan Klinedinst, Delia Graff, Gilbert Harman, Irene Heim, Stephen Neale, James Pryor, and Philippe Schlenker for useful comments; none of them are responsible for any errors. I'm also very grateful to the UCL Pragmatics Reading Group, UCLA Semantics Lunch, as well as SALT 16, of course, for being very helpful and patient audiences.

${ }^{1}$ Weak NPIs do not need to be licensed locally, but they cannot be unlicensed by higher-up operators. Thus, they only need to be in some local downward-entailing context to be licensed. I will discuss intervention later.

${ }^{2}$ I will discuss some exceptions in Section 1.5. 
the same reasons; nonetheless, any account of weak-NPI licensing should be able to say something about the full range of non-monotonic NPI-licensors.

- Antecedents of conditionals:

(2) If John had walked on any weeds he would have tracked dirt into the house.

- Restricter of quantifiers such as "most":

(3) Most men with any revolutionary commitments were executed.

- Unfocused contexts with "only"

(4) Only John did any work.

- Exactly $n$ (judgments here are much less consistent than with the previous cases)

(5) Exactly three people with any money showed up.

(6) Exactly three people did any work at all. ${ }^{3}$

We will later cover some other examples, but for now this gives some sense of the range of examples where apparently non-monotonic contexts allow NPIs.

\subsection{Strawson Downward-Entailment}

I will begin by reviewing one of the most prominent proposals for expanding the downward-entailment criterion to include some non-monotonic cases. This is von Fintel's proposal that rather than use downward-entailment we should use Strawson-downward-entailment (von Fintel 1999). A sentence $S$ Strawson-entails $S^{\prime}$ just in case in all cases where the presuppositions of both $S$ and $S^{\prime}$ are satisfied the truth of $S$ implies the truth of $S^{\prime}$.

One problem for this account is the case of "most." As in (7), "most" allows NPIs in its restricter predicate.

(7) Most student with any money came.

But in this example the context where "any" appears do not seem to be StrawsonDE since the only presupposition plausibly triggered by "most" is the existence of something satisfying the restricter. So the Strawson-DE proposal does not seem to handle this case.

One way to make the context after "most" Strawson-DE is to add extra conversational presuppositions. For instance, "any" would be in a Strawson-DE context in (7) if we also presuppose that, if most students with amount $x$ of money came,

\footnotetext{
${ }^{3}$ All of these cases are discussed in the literature see, e.g., Linebarger (1980), Heim (1987), and von Fintel (1999).
} 
then most students with amount $y>x$ also came. ${ }^{4}$ This proposal, however, would allow even upward-entailing contexts to allow NPIs if the right presuppositions are in play. Consider this example:

A student with any money came.

If we presuppose that if a student with amount $x$ of money came then a student with amount $y>x$ also came then the context after "a" in (8) should allow NPIs. However, with "a," unlike with "most," the NPI is plainly unacceptable. If Strawson DE is the right criterion for NPI licensing and such extra non-grammatical presuppositions are allowed we should expect uses of NPIs in UE contexts not to be infelicitous but rather to require somewhat unusual, but not impossible, presuppositions. ${ }^{5}$

In addition, it is not clear that an utterance of (7) actually requires that the presupposition I suggested be present. So adding extra presuppositions to the context to explain why sentences like (7) allow NPIs both over-generates and seems to go against intuitions of what is actually presupposed when NPIs are used.

Another possible explanation for why "most" licenses NPIs is that it includes a hidden generic operator. In his paper, von Fintel (1999) gives a plausible account of why generics and conditionals license NPIs in their restricters, so if there is a hidden a generic operator in sentences such as (7) then the Strawson-DE account makes the correct prediction. However, it is not clear that sentences with "most" and NPIs always make counterfactually-supporting generic statements. One can easily say for instance:

(9) Most people in the group who'd ever been to France happened to be Japanese.

There is no intuitive sense that any generalization is being made, but the NPI is acceptable.

The Strawson-DE account also fails to explain why uses of "exactly" allow NPIs, as in (5) and (6). In these sentences, the contexts are neither DE nor StrawsonDE but they do allow NPIs (with some variation in judgments between speakers).

Strawson-DE thus seems to be an insufficient weakening of the DE criterion for NPI licensing. It explains some cases well, such as why "only" allows NPIs, but it does not handle "most" or "exactly" convincingly.

\footnotetext{
${ }^{4}$ This closely follows the proposal of Heim (1987) for dealing with the problem of "most." There are some complications about exactly how we define the context that "any" appears in, and what counts as a legitimate strengthening of the predicate in that context.

${ }^{5}$ Such presuppositions may not be so plausible with students, but they can be plausible in mathematical cases. One can presuppose, for instance, of a set that if two numbers whose square roots are greater than $n$ are in it, than two number whose square roots are greater than $n+1$ are in it. This does not make it acceptable to say the following, however:
}

(i) ? Two numbers with any square roots are in this set. 


\subsection{Covert "Only"}

Another proposal to explain the appearance of NPIs in various non-monotonic contexts is to argue for the presence of a covert "only" around such contexts. In itself this is not a complete proposal since one also needs to explain why "only" can license NPIs. For convenience I'll assume this proposal is just an extension of the Strawson-DE account, since that account yields a particularly elegant treatment of "only."

An interesting piece of evidence has been put forward by Nishiguchi (2005) in an attempt to explain non-monotonic NPI-licensing in terms of covert "only." Her claim is that there can be a covert "only" only where an overt "only" could grammatically appear. So, for instance, consider this pair:

(10) Only exactly three percent of the people made it.

(11) ? Only exactly ninety-nine point nine percent of the people made it.

There are certain contexts in which (10) would be acceptable but (11) would not be. In general "only" can only associate with contextually low scalars, not high ones. Nishiguchi suggests that this explains the different judgements reported with different exact numerals:

(12) Exactly three people did any work.

(13) ? Exactly five hundred people did any work.

Where five hundred is construed as a high scalar number, it seems that only the first of these pairs is acceptable. (I'll discuss this contrast again in Section 1.5.)

Despite this nice piece of data, postulating a covert "only" is not very helpful for explaining the distribution of NPIs. This is because the proposal does not explain why upward-entailing quantifiers do not license NPIs.

(14) Only a few people brought any money

(15) ? A few people brought any money.

As (14) and (15) show, the possible presence of an overt "only" does not mean that an NPI is allowed. So without a substantive account of the distribution of covert "only," which must differ from overt "only," this proposal is non-predictive.

\subsection{Nonveridicality}

Another major proposal for explaining the presence of NPIs in non-monotonic environments is by use of the criterion of nonveridicality (Giannakidou 2002).

There are different definitions of nonveridicality for different semantic contexts. I will focus on quantifiers. The relevant definition Giannakidou gives is that a restricter position of a quantifier is nonveridical if the entire sentence does not entail the existence of something satisfying the predicate in the restricter position:

(Non)veridicality of determiners and quantifiers: A determiner/quantifier $d$ is veridical w.r.t. its NP argument iff it holds 
that: $\llbracket d \mathrm{NP} \mathrm{VP} \rrbracket=1 \rightarrow \llbracket \mathrm{NP} \rrbracket \neq \emptyset$; otherwise, $\mathrm{d}$ is nonveridical. (Giannakidou 2002: Definition 5)

This definition is too strong for quantifiers like "every" which generally lead to an existence entailment but still license NPIs:

(16) Every student with any health problems went to the nurse.

This sentence seems to imply that at least one student had a health problem, and "any" would seem to be under a nonveridical determiner, "every." The facts about such examples are apparently different for modern Greek, as reported by Giannakidou, but the proposal does not seem to work for English.

Now, there may be ways of tweaking the definition of nonveridicality to cover these cross-linguistic differences. ${ }^{6}$ But there is still a basic problem with this whole effort. The problem is that nonveridicality is too broad a feature to account for the distribution of weak NPIs in English. For nonveridicality is meant to unify the licensing conditions of both free-choice and NPI "any" (Giannakidou 2002). If it does that however, it will not explain the distribution of other weak NPIs like "ever" which cannot appear in certain nonveridical environments, as shown by this contrast:

(17) I might meet anybody.

(18) ? I might ever come to the meeting.

Thus, we would need some restriction on nonveridicality that covers the distribution of "ever" in non-monotonic context without including all the nonveridical contexts. We have seen that simple DE-ness or Strawson-DEness will not do. Likewise, nonveridicality will not do since it does not provide a predictive account of the distribution of "ever" as opposed to "any."

\subsection{Strengthening and Weakening Explanations}

Kadmon and Landman (1993) set a new standard in the study of NPIs by trying to explain not just where NPIs are licensed but also why they are licensed exactly where they are. Their story was written with "any," in mind, but it can be easily extended to handle other weak NPIs such as "ever" or "at all."7

A version of their basic story goes as follows: NPIs are exceptionally low scalar items. When an NPI like "any" is used, the specificity of the domain of possible referents is taken to be as low as possible (so the domain is "widened" in their terminology). When "ever" is used the specificity of the time the event took place (or perhaps the number of times it took place) is as low as possible. In addition to calling for low scalar values, NPIs also must participate in a strengthening of the statement being made by virtue of being a low-valued scalar. ${ }^{8}$. So NPIs need

\footnotetext{
${ }^{6}$ Some suggestions are given by Giannakidou (2004).

${ }^{7}$ Due exception, of course, needs to be made for fact that "ever" and "at all" do not have freechoice uses. For an approach to this problem see Horn (2000).

${ }^{8}$ This must be checked recursively; for a proposals along the lines of Kadmon and Landman that work recursively, see Chierchia (2004) and Krifka (1995).
} 
to appear under negation or some other operator that has the semantic effect of reversing what is normally a weakening of a statement into a strengthening.

This seems to me a pretty good story. One objection raised by Krifka (1995) is that normal uses of NPIs do not seem to call for weaker scalar properties. For instance, it is hard to see how "any integer" is weaker than "an integer." However, this worry does not seem very serious. It may be that certain calls for a weakening have no truth-conditional effect, since there is no room for widening the domain. That does not mean that these sorts of NPIs are not characterized by being at the lowest end of a scale.

However, Kadmon and Landman's account predicts that NPIs can only appear in (locally) DE environments. This is because of the fact that for a weakening of a predicate to result in a logically stronger statement the context has to be strictly downward entailing. This was meant to be one of the virtues of the account, but it is also a weakness since non-monotonic contexts also license NPIs.

A very simple alteration of Kadmon and Landman's account will handle this problem. All we need to say is that for an NPI to be acceptable the weaker scalar item must lead, not to a stronger statement, but to a statement that is not weaker. If this is our criterion than NPIs will be allowed in all contexts that are DE or non-monotonic. ${ }^{9}$

The point of this diversion is to show that the proposal that NPIs are allowed in non-monotonic and DE environments fits in just as well with Kadmon and Landman's elegant treatment of NPI meaning and licensing as the proposal that NPIs are allowed in just DE environments does. All that is needed is to replace Kadmon and Landman's strengthening criterion with a not-weaken criterion. I want to suggest that the best generalization about weak NPIs in English is that they are allowed in any environment that is non-monotonic or downward-entailing.

\subsection{Exceptions}

I imagine there may be some skepticism about the idea that the criterion of NPIlicensing (in English) is simply being in either a DE or non-monotonic context. I have tried to indicate that the usual conservative extensions of the notion of DEness are inadequate to cover the full range of NPI licensing. However, the effort expended on expanding the notion of DEness to cover more cases was motivated by reluctance to adopt the hypothesis that non-monotonic contexts license NPIs. ${ }^{10}$

I can think of two reasons for denying that the conditions of NPI licensing in English are simply being in a non-monotonic or DE environment. One is that there are certain non-monotonic contexts in English that do not license NPIs. The other is that in other languages NPI licensing is more strict. I lack the expertise to deal with cross-linguistic licensing conditions, so I will just discuss the examples of non-monotonic contexts that do not license NPIs.

\footnotetext{
${ }^{9}$ In related work, I define contexts that are not-UE in terms of what I call domain-sensitivity (Rothschild 2005).

${ }^{10}$ This hypothesis has been adopted by some authors, such as Linebarger (1980), Neale (2000).
} 
One such context, mentioned above, is high numerals combined with "exactly":

? Exactly two million people have ever been to this forest.

The idea, which some speakers confirm, is that if two million is regarded as an unexpectedly high number, than the NPI "ever" is infelicitous. This may be an example of a non-monotonic context failing to license an NPI, but it is not a problematic case for the hypothesis that NPIs are generally licensed in DE or non-monotonic contexts. For any account of NPI-licensing needs to deal with intervention effects. The basic pattern is as follows: whenever we have an operator that creates an NPIlicensing environment, if an intervener appears between that operator and the NPI then the NPI will not be licensed. Here is the classic example:

? I doubt that every one drank any coke.

Here the NPI-licensor "doubt" fails to license "any" because of the intervention of "every." I do not know how to account for intervention effects, but they do not show that the basic semantic condition for NPI-licensing is in terms of DEness rather than a looser condition.

A more troubling example, brought to my attention by Philippe Schlenker, involves the word "odd" (or "even").

? An odd (even) number of students did any work.

It is clear that "any" is in a non-monotonic context here but that, nonetheless, it is infelicitous. I think there are two sorts of move one can make in the face of this sort of example. The first is to suggest that NPI-licensing is determined by a modularized system that is not sensitive to the meanings of all the individual predicates. The meaning of "odd" requires mathematical sophistication to master, and it is not surprising that the modularized NPI-licensing system does not respond to it. ${ }^{11}$

The second move is to say that the condition of NPI licensing is not that the lower scalar value of the NPI word needs to result in a statement that is logically independent of a statement with a higher scalar value (as I suggested above). Rather it needs to result in a statement that is more unexpected than a statement with a higher scalar value. One can imagine a context in which it is more unexpected that John didn't eat one apple than that he didn't eat two apples or a context in which it is more unexpected that most people with any money went to the cinema rather than most people with over three dollars went to the cinema. However, it is hard to

\footnotetext{
${ }^{11}$ It seems to me that for this very reason certain downward-entailing contexts will not license NPIs. Consider, for instance the concept of being a self-squared number: a number that its own square. The only two self-squared numbers I can think of are 0 and 1 . So a "self-squared number" just means a non-negative integer less than two. This should create certain DE contexts, as in this example:
}

(ii) A self-squared number of students did any work.

The NPI "any" seems quite odd here even though it is clearly in a DE environment. My only claim above is that "odd" in (21) acts a bit like "self-squared" here. 
imagine that it could be more unexpected that an odd number of students did any work, than it would be unexpected that an odd number of students did at least one hours worth of work. This proposal would need to be worked out in more detail, but it provides some hope of giving a principle explanation of why most non-monotonic contexts license NPIs but words like "odd" and "even" do not.

There are more exceptional cases that could be discussed, but all I want to make plausible is that there are reasonable things to say about many of these cases.

\section{Applications}

One of the motivations for getting a correct account of NPI licensing is that facts about NPIs can be used to make inferences about the semantics of various construction. What exactly one says about NPIs has a huge influence on what says about the semantics and pragmatics of constructions with comparatives, superlatives, "only," and, I shall argue here, scalar implicatures and singular definite descriptions.

I will suggest that the generalization I have supported above, that English allows NPIs in non-monotonic contexts, provides a challenge for certain accounts of scalar implicature calculation as well as certain semantics for the singular definite article.

\subsection{Scalar implicature}

It is well-known that scalar implicatures lead to certain forms of non-monotonicity. Consider the following sentence:

(22) Five people drank coke.

An utterance of (22) in normal circumstances implicates a DE statement, (23):

(23) No more than five people drank coke.

The two statements together put "drank coke" in a non-monotonic context.

On the standard Gricean story this is no problem. For conversational implicatures occur independently of syntactic and semantic processing. They are inferences about the speaker's intent on the assumption the speaker is following certain maxims or rules of conversation. We should not expect, then, the non-monotonicity of scalar implicatures to affect NPI-licensing.

However, various problems with the Gricean account have been noted which have led some theorists to think scalar implicatures are actually marked syntactically in the grammar (Chierchia 2004, Fox and Hackl 2005). Here is one typical statement from Uli Sauerland (2004):

I follow Krifka (1995), Fox (2003), King \& Stanley (2004) to assume that the points at which embedded implicatures are computed are marked by an implicature computation operator that is projected in the syntax. 
There are various different ways in which such a proposal can be fleshed out. However, it seems likely that any proposal that adequately captures the nonmonotonicity of statements with scalar implicatures will posit an operator in the syntax that has the effect of creating a non-monotonic environment in the semantics.

On the face of it, then, such a proposal will lead to a prediction, namely that scalar implicatures, such as that in (22), will license NPIs. However, this prediction is not borne out:

? Five people drank any coke.

I am not suggesting that this is an insurmountable problem for grammaticalized accounts of scalar implicatures. However, those proposing such accounts need to explain why operators that do the work of scalar implicatures do not license NPIs. One cannot merely resort to saying that such operators do not create DE environments or Strawson DE environments, since those accounts fail exactly at predicting that non-monotonic contexts $d o$ license NPIs. ${ }^{12}$

\subsection{Singular Definite Descriptions}

My second example to which we can apply facts about NPI licensing to make inferences about the semantics and pragmatics is the case of singular definite descriptions. What makes singular definite descriptions interesting in this respect is their famous uniqueness implications (Russell 1905). Russell's original proposal was to treat "the" in such a way as to make the following two sentences equivalent:

The King of France is bald.

(26) There is at least one King of France, there is no more than one King of France, and whoever is a King of France is bald.

The neo-Russellian account takes "the" to be generalized quantifier whose semantics make the equivalence come out (Neale 1990).

This neo-Russellian proposal, while popular within the philosophy of language, is not the dominant view within linguistic semantics. Rather the most standard view is the so-called Fregean view, according to which definite descriptions only presuppose uniqueness, rather than semantically encoding it. ${ }^{13} \mathrm{~A}$ third, and less popular, view of definite descriptions takes them to be expressions that neither semantically encode nor presuppose uniqueness, but rather are used to pick out a familiar discourse referent, if there is one. ${ }^{14}$ On this type of view any uniqueness implications that arise from the use of a definite description must come as a conversational implicature.

\footnotetext{
${ }^{12}$ This problem does not seem to affect Krifka (1995) since NPI licensing and scalar-implicature calculation occur in tandem on his account.

${ }^{13}$ Specifically, the Fregean view takes the definite description "the F" to be an $e$-type expression that refers to the unique $\mathrm{F}$ if there is one, and is undefined otherwise.

${ }^{14}$ This type of view comes in a wide variety of forms, including Heim's file-card semantics, and recent proposals by Roberts (2003) and Szabó (2000).
} 
These three views are not exhaustive, but they will do:

- Russellian view: "the F is G" = "there is exactly one F and it is G." (Neale 1990)

- Fregean view: uniqueness presupposed. (Heim and Kratzer 1998)

- Familiarity view: uniqueness implications (when they occur) are conversationally implicated. (Heim 1982, Roberts 2003, Szabó 2000)

What I will argue for in the rest of this paper is that the data on NPI licensing I reviewed above suggests that the Russellian view is incorrect as well as providing some evidence against the Fregean view.

\subsubsection{Russell}

The basic problem with the Russellian view, is that it makes definite descriptions non-monotonic in their restricter position. This is easy to see:

(27) The tall man came.

(28) The man came.

(29) The very very tall man came.

On the Russellian semantics (27) neither entails (28) nor (29) so that the predicate inside the definite description is in a non-monotonic environment. In addition, on the Russellian view it is very possible in some contexts that (28) could be more unexpected than (27) since it could be more surprising that there is just one man in the room than that there is just one tall man in the room. So we should expect an NPI to be allowed inside a definite description. However, NPIs are not allowed inside singular definite descriptions:

(30) ? The man with any money came to the casino.

What is even more interesting is that when we make explicit some form of uniqueness, then an NPI is allowed in the definite description:

(31) The one/only/sole man with any money came to the casino

This last fact is exactly what we should expect given that non-monotonic environments allow NPIs in English, though it is unexpected if we suppose that only DE environments allow NPIs. ${ }^{15}$

\footnotetext{
${ }^{15}$ Note that there is some difference between "the one" and "the only": "the one" presupposes uniqueness, "the only" seems to assert it. This can be seen by looking at projection over negation:

(iii) John is not the only man to have come here.

(iv) John is not the one man to have come here.
} 


\subsubsection{Frege}

On the Fregean view uniqueness is presupposed rather than asserted. I have made no predictions so far about whether lexically-trigger non-monotonic presuppositions license NPIs. All I want to point out is that there is some evidence that they do.

Two expressions that seems to make non-monotonic presuppositions are "the one" and "both". Both of these expressions sometimes license negative polarity items in their restricter predicates:

(32) In the study, both dogs that received any physical therapy eventually managed to walk again.

(33) In the study, the one dog that received any physical therapy eventually managed to walk.

If the Fregean view were correct we might expect singular definite descriptions to also license negative polarity items in similar examples. However, in the same contexts informants consistently report that an NPI is not acceptable:

? In the study, the dog that received any physical therapy eventually managed to walk again.

This provides a limited piece of evidence that the Fregean view is not correct. However, while judgments about (34) are relatively clear, judgments about (32) are rather variable. Moreover, in (33) there seems to be space between "the" and "one" where a different operator could lead to the NPI licensing (though it is hard to see what that operator would be).

\subsubsection{Uniqueness as a Conversational Implicature}

My last point is to note that while the Russellian account has trouble with NPIs and the Fregean account may have some also, the familiarity-style accounts are compatible with the data on NPI licensing. For on these accounts definite descriptions do not make presuppositions of uniqueness. Rather they are used to pick out familiar discourse referents. One can sometimes infer from the conversational context that there is only one entity satisfying the description, but that inference is not grammatically encoded. In this case, singular definite descriptions do not appear to be non-monotonic in their restricter, and so we should not expect them to license NPIs.

\subsection{NPIs as Evidence for Pragmatic Effects}

In the case of scalar implicatures and the case of singular definite descriptions there is evidence that certain types of monotonicity are not grammatically encoded. This is because grammatically encoded non-monotonicity generally licenses NPIs, but in these constructions NPIs are not licensed. So the lack of NPI licensing in singular definite descriptions and scalar implicatures is evidence that certain aspects of their 
reported meaning may be due to genuine pragmatic inferences not present in the syntax or semantics.

\section{References}

Chierchia, Gennaro: 2004, 'Scalar Implicatures, Polarity Phenomenon, and the Syntax/Pragmatic Interface', in A. Belleti (ed.), Structures and Beyond. Oxford University Press, Oxford, UK.

von Fintel, Kai: 1999, 'NPI-Licensing, Strawson-Entailment, and ContextDependency', Journal of Semantics 16, 97-148.

Fox, Danny and Martin Hackl: 2005, 'The Universal Density of Measurement'. Manuscript.

Giannakidou, Anastasia: 2002, 'Licensing and Sensitivity in Polarity Items: From Downward Entailment to (Non)Veridicality', Chicago Linguistics Society 38.

Giannakidou, Anastasia: 2004, 'How Much Can We Weaken Downward Entailment?'. Unpublished manuscript.

Heim, Irene: 1982, The Semantics of Definite and Indefinite Noun Phrases, $\mathrm{PhD}$ dissertation, University of Massachusetts, Amherst.

Heim, Irene: 1987, 'A Note on Negative Polarity and Downward-Entailingness', NELS 14, 98-107.

Heim, Irene and Angelika Kratzer: 1998, Semantics in Generative Grammar. Blackwell.

Horn, Larry: 2000, 'Any and Ever: Free Relatives and Free Choice', IATL 14, 71-111.

Kadmon, Nirit and Fred Landman: 1993, 'Any', Linguistics and Philosophy 4.2, 279-298.

Krifka, Manfred: 1995, 'The Semantics and Pragmatics of Polarity Items', Linguistic Analysis 25, 209-257.

Linebarger, Marcia C: 1980, The Grammar of Negative Polarity, PhD dissertation, MIT.

Neale, Stephen: 1990, Descriptions. MIT Press, Cambridge, MA.

Neale, Stephen: 2000, 'Persistence and Polarity', in U. Egli and K. von Heusinger (eds.), Reference and Anaphoric Relations. Kluwer, Munich.

Nishiguchi, Sumiyo: 2005, 'Covert "Only" and NPI Licensing'. Handout for 79th LSA.

Roberts, Craige: 2003, 'Uniqueness in Definite Noun Phrases', Linguistics and Philosophy 26, 287-350.

Rothschild, Daniel: 2005, 'Domain-Sensitivity and Negative Polarity'. Unpublished manuscript.

Russell, Bertrand: 1905, 'On Denoting', Mind 14, 479-493.

Sauerland, Uli: 2004, 'On Embedded Implicatures', Journal of Cognitive Science 5. 
Szabó, Zóltan: 2000, 'Descriptions and Uniqueness', Philosophical Studies 101, 29-57. 